BMJ Open

Diabetes

Research

\& Care

\title{
Selenium supplementation and insulin resistance in a randomized, clinical trial
}

\author{
Elizabeth Theresa Jacobs, ${ }^{1,2,3}$ Peter Lance, ${ }^{2,4}$ Lawrence J Mandarino, ${ }^{4,5}$ \\ Nathan A Ellis, ${ }^{2} \mathrm{H}-\mathrm{H}$ Sherry Chow, ${ }^{2}$ Janet Foote, ${ }^{1}$ Jessica A Martinez, ${ }^{2,3}$ \\ Chiu-Hsieh Paul Hsu, ${ }^{1,2}$ Ken Batai, ${ }^{6}$ Kathylynn Saboda, ${ }^{2}$ Patricia A Thompson ${ }^{7}$
}

To cite: Jacobs ET, Lance P, Mandarino LJ, et al. Selenium supplementation and insulin resistance in a randomized, clinical trial. BMJ Open Diab Res Care 2019;7:e000613. doi:10.1136/ bmjdrc-2018-000613

ETJ and PL contributed equally.

Received 28 August 2018 Revised 13 December 2018 Accepted 22 December 2018

Check for updates

(C) Author(s) (or their employer(s)) 2019. Re-use permitted under CC BY-NC. No commercial re-use. See rights and permissions. Published by BMJ.

${ }^{1}$ Department of Epidemiology and Biostatistics, Mel and Enid Zuckerman College of Public Health, University of Arizona, Tucson, Arizona

${ }^{2}$ University of Arizona Cancer Center, Tucson, Arizona

${ }^{3}$ Department of Nutritional

Sciences, University of Arizona, Tucson, Arizona

${ }^{4}$ Department of Medicine, University of Arizona, Tucson, Arizona

${ }^{5}$ Center for Disparities in Diabetes, Obesity and Metabolism, University of Arizona, Tucson, Arizona ${ }^{6}$ Department of Surgery, University of Arizona, Tucson, Arizona

${ }^{7}$ Department of Medicine, Stony Brook University, New York City, New York, USA

Correspondence to Dr Elizabeth Theresa Jacobs; jacobse@email.arizona.edu

\section{ABSTRACT}

Objective While controversial, observational and randomized clinical trial data implicate the micronutrient selenium (Se) in the development of type 2 diabetes (T2D). The aim of this study was to test the hypothesis that Se supplementation adversely affects pancreatic $\beta$-cell function and insulin sensitivity.

Research design and methods In a subset of 400 individuals participating in a randomized, placebocontrolled trial of Se at $200 \mu \mathrm{g} /$ day for colorectal adenomatous polyps, fasting plasma glucose and insulin were measured before randomization and within 6 months of completing intervention. Change in the homeostasis model assessment- $\beta$ cell function (HOMA2- $\% \beta$ ) and insulin sensitivity (HOMA2-\%S) were compared between arms. A subgroup of 175 (79 Se and 96 placebo) participants underwent a modified oral glucose tolerance test (mOGTT) at the end of intervention and change in glucose values was assessed.

Results No statistically significant differences were observed for changes in HOMA2- $\% \beta$ or HOMA2- $\%$ S between those who received Se compared with placebo. After a mean of 2.9 years on study, mean HOMA2- $\% \beta$ values were $3.1 \pm 24.0$ and $3.1 \pm 29.8$ for the Se and placebo groups, respectively $(p=0.99)$. For HOMA2- $\% S$, the values were $-0.5 \pm 223.2$ and $80.9 \pm 1530.9$ for the Se and placebo groups, respectively $(p=1.00)$. Stratification by sex or age did not reveal any statistically significant effects on insulin sensitivity by treatment group. For mOGTT, mean baseline fasting blood glucose concentrations were significantly higher among participants in the placebo group compared with the Se group $(96.6 \pm 14.6$ and $92.3 \pm 12.0$, respectively; $\mathrm{p}=0.04$ ), a trend which remained through the $20 \mathrm{~min}$ assessment.

Conclusions These findings do not support a significant adverse effect of daily Se supplementation with $200 \mu \mathrm{g} /$ day of selenized yeast on $\beta$-cell function or insulin sensitivity as an explanation for previously reported associations between Se and T2D. Further clarification of longer term effects of Se is needed.

Clinical trial registry NIH Clinical Trials.gov number NCT00078897.

\section{INTRODUCTION}

The trace element selenium (Se) has antioxidant physiological functions that were proposed to potentially reduce the risk for cancer. ${ }^{1-3}$ This hypothesis was tested in large, randomized clinical trials (RCTs) of Se and

\section{Significance of the study}

What is already known about this subject?

- A high proportion of adults in the USA consume dietary supplements, including selenium.

- Selenium supplementation has been associated with the development of type 2 diabetes in some studies.

What are the new findings?

- After 2.9 years of selenium supplementation with $200 \mu \mathrm{g} /$ day, selenium had no effect on insulin sensitivity or $\beta$-cell function as compared with the placebo group.

- Stratification by sex and age revealed no effect modification in response to selenium.

How might these results change the focus of research or clinical practice?

- These results provide key information regarding a lack of adverse effect of selenium supplementation on insulin sensitivity in a clinical trial.

- This work does not support a major role for selenium in insulin sensitivity or $\beta$-cell function.

- These results provide key information for clinicians to convey to patients as the use of dietary supplements continues in a large proportion of adults in the USA.

skin and prostate cancers and colorectal adenoma, ${ }^{1-3}$ with equivocal results. Secondary analyses were conducted within the Nutritional Prevention of Cancer (NPC) trial of skin cancer prevention, where participants receiving Se had an increased risk for type 2 diabetes (T2D) as compared with those receiving placebo. ${ }^{4}$ Later clinical trials, specifically the Selenium and Vitamin E Cancer Prevention Trial (SELECT) and the Selenium Trial, showed no statistically significant overall increased risk for T2D risk after supplementation with $200 \mu \mathrm{g} /$ day Se as compared with placebo. ${ }^{23}$ In contrast, results from observational studies have generally demonstrated an increased risk for T2D among those with higher blood or toenail Se concentrations. ${ }^{5-9}$ However, these results are challenging to interpret because of marked geographical 
variation in dietary intake of Se, which in turn elicits major differences in circulating concentrations of Se. In addition, the mechanism(s) whereby Se supplementation might lead to insulin resistance has yet to be determined, though there are several viable hypotheses.

At one time, Se was thought to be beneficial in relation to glucose homeostasis, having been shown in rodent models to have insulin mimetic effects ${ }^{10}$ and reduce insulin resistance. ${ }^{11}$ However, the agent used in these earlier experimental studies was selenate, an inorganic form of SE that is present in only small amounts in the human diet. More recently, the focus of human research on the biological effects of ingested SE has generally been on selenoproteins, a family of proteins that incorporate the amino acids selenomethionine and selenocysteine and mediate the actions of Se. ${ }^{12}$ One such selenoprotein is glutathione peroxidase-1 (GPx-1), which has an important role in protection against oxidative damage. ${ }^{12}{ }^{13}$ In addition to its physiological role, experiments with mouse models have demonstrated that GPx-1 overexpression results in hyperglycemia and hyperinsulinemia; consistent with this adverse effect, GPx-1 null animals exhibited better insulin sensitivity compared with wild-type mice. ${ }^{1415}$

Because of the equivocal results for the impact of Se on T2D and insulin action, we sought to determine the effect of Se supplementation on $\beta$-cell function and insulin sensitivity by employing the homeostatic model assessment of $\beta$-cell function (HOMA2-\% $\beta$ ) and insulin sensitivity (HOMA2-\%S), and oral glucose tolerance tests (OGTT) as the primary endpoints. We addressed this objective within the context of the Selenium Trial, a randomized, placebo-controlled clinical trial of the effect of selenized yeast for prevention of colorectal adenomas. ${ }^{3}$

\section{RESEARCH DESIGN AND METHODS}

\section{Study population}

Participants for this study were drawn from the Selenium Trial (NIH Clinical Trials.gov number NCT00078897). The Selenium Trial has been described in detail previously. ${ }^{3}{ }^{16}$ Briefly, this was a double-blind, placebo-controlled trial where participants were randomized to receive either $200 \mu \mathrm{g} /$ day of Se as selenized yeast or placebo to assess the effect of Se on the development of metachronous (ie, new or 'recurrent') colorectal adenomas. Participants in the trial were men and women between the ages of 40 and 80 years recruited from endoscopy clinics in Arizona, Colorado, Texas, and New York, with one or more colorectal adenomas of diameter $\geq 3 \mathrm{~mm}$. All trial participants were required to have undergone total colonoscopy and complete removal of all colorectal adenomas within the 6 months before registration. Management of study data and biospecimens was conducted at the University of Arizona Cancer Center (Tucson, Arizona, USA). ${ }^{16}$

A total of 400 participants were selected for a substudy using HOMA2 assessments. Participants were identified based on the availability of a baseline fasting blood draw, as well as a postintervention fasting blood draw within 6 months of intervention completion. Exclusion criteria included having taken celecoxib before this arm of the original protocol of the Selenium Trial was discontinued as directed by the Food and Drug Administration, ${ }^{316}$ and not having a fasting blood draw both preintervention and postintervention. A stratified random sample of available individuals was then selected from the eligible participants, based on year of randomization, leaving 195 from the placebo group and 205 from the Se treatment group for inclusion in the present analyses. Of these participants, results for HOMA2 assessments were available for 188 participants in the placebo group and 201 in the Se group. Based on a two-sample t-test, a sample size of 389 allows us to obtain a power of $81 \%$ detecting an effect size of 0.29 Cohen's d for changes in HOMA parameters between the placebo and Se groups at a significance level of $5 \% .{ }^{17}$ For the modified OGTT (mOGTT) study, a convenience sample of 175 participants was selected. All participants who remained on the trial near the end of follow-up were approached and asked if they would like to take part in the mOGTT substudy. Individuals who agreed to participate were then scheduled for the test, including 96 participants in the placebo group and 79 from the intervention group. The conduct of the trial was in accordance with requirements of the local IRB at each study site.

\section{Exposure and outcome assessment}

To assess the primary endpoints of $\beta$-cell function (HOMA2-\% $\beta$ ) and insulin sensitivity (HOMA2-\%S), we used the University of Oxford Diabetes Trials Unit HOMA2 calculator Application Programming Interface downloaded from http://www.dtu.ox.ac.uk/homacalculator/index.php. Fasting blood draws from participants at the end of the Selenium Trial were obtained. ${ }^{18}$ As described above, a total of 400 participants were selected from the Se intervention and placebo groups to ascertain if there were differences between them for insulin sensitivity and $\beta$-cell function. For plasma glucose concentrations, blood was drawn into a tiger top tube at each of the time points, processed and shipped directly to a commercial laboratory (Sonora Quest, Tempe, Arizona, USA). Assessment of plasma insulin concentrations at each time point were determined in the laboratory of Dr Lawrence Mandarino at Arizona State University (Tempe, Arizona, USA) using immunoassays Alpco Immunoassays (Salem, New Hampshire, USA). Each sample from an individual was run in the same assay batch to avoid laboratory drift, along with five internal reference standards included in each batch. The coefficients of variation for glucose and insulin were less than $5 \%$.

While HOMA2 measures are an efficient strategy for assessing insulin resistance in large epidemiological studies, they are unable to capture dynamic changes in insulin secretion and do not directly address functionality. ${ }^{19}$ We therefore selected a subset of the clinical trial 
Table 1 Baseline characteristics of homeostatic model assessment study participants

\begin{tabular}{|c|c|c|c|c|}
\hline Characteristic & Placebo (N = 195) & Selenium $(N=205)$ & Total $(\mathrm{N}=400)$ & P value* \\
\hline \multicolumn{5}{|l|}{ Age } \\
\hline Mean (SD) & $62.6(8.5)$ & $63.4(9.3)$ & $63.0(8.9)$ & 0.32 \\
\hline \multicolumn{5}{|l|}{ Body mass index } \\
\hline Mean (SD) & $29.2(4.9)$ & $29.2(5.4)$ & $29.2(5.2)$ & 0.99 \\
\hline Mean (SD) & $107.8(63.0)$ & $101.7(56.1)$ & $104.6(59.5)$ & \multirow[t]{2}{*}{0.32} \\
\hline Median (Q1, Q3) & $93.7(70.5,128.9)$ & $88.8(65.1,124.4)$ & $91.1(67.9,126.0)$ & \\
\hline \multicolumn{5}{|c|}{ Selenium supplemental intake ( $\mu \mathrm{g} /$ day) } \\
\hline Mean (SD) & $7.7(11.7)$ & $5.5(7.2)$ & $6.5(9.7)$ & 0.03 \\
\hline Median (Q1, Q3) & $134.4(121.7,152.1)$ & $136.0(124.0,155.5)$ & $134.6(122.6,153.8)$ & 0.16 \\
\hline \multicolumn{5}{|l|}{ Gender } \\
\hline Female & $62(31.8 \%)$ & $74(36.1 \%)$ & $136(34.0 \%)$ & \multirow[t]{2}{*}{0.36} \\
\hline Male & $133(68.2 \%)$ & $131(63.9 \%)$ & $264(66.0 \%)$ & \\
\hline \multicolumn{5}{|l|}{ Ethnicity } \\
\hline Non-Hispanic & $186(95.4 \%)$ & $192(93.7 \%)$ & $378(94.5 \%)$ & \multirow[t]{2}{*}{0.45} \\
\hline Hispanic & $9(4.6 \%)$ & $13(6.3 \%)$ & $22(5.5 \%)$ & \\
\hline \multicolumn{5}{|l|}{ Race } \\
\hline Native American & $0(0.0 \%)$ & $2(1.0 \%)$ & $2(0.5 \%)$ & 0.21 \\
\hline Some college & $55(28.2 \%)$ & $78(38.2 \%)$ & $133(33.3 \%)$ & \multirow{3}{*}{0.04} \\
\hline College degree & $35(17.9 \%)$ & $36(17.6 \%)$ & $71(17.8 \%)$ & \\
\hline Postgraduate & $57(29.2 \%)$ & $48(23.5 \%)$ & $105(26.3 \%)$ & \\
\hline \multicolumn{5}{|c|}{ Cigarette smoking status } \\
\hline Current smoker & $17(8.9 \%)$ & $22(10.8 \%)$ & $39(9.9 \%)$ & \multirow[t]{3}{*}{0.5} \\
\hline Previous smoker & $91(47.6 \%)$ & $104(51.2 \%)$ & 195 (49.5\%) & \\
\hline Never smoker & $83(43.5 \%)$ & $77(37.9 \%)$ & $160(40.6 \%)$ & \\
\hline \multicolumn{5}{|l|}{ Diabetes } \\
\hline No diabetes & $176(90.3 \%)$ & $193(94.1 \%)$ & 369 (92.3\%) & \multirow[t]{2}{*}{0.15} \\
\hline Has diabetes & $19(9.7 \%)$ & $12(5.9 \%)$ & $31(7.8 \%)$ & \\
\hline \multicolumn{5}{|c|}{ Personal history of cancer } \\
\hline Never had cancer & 185 (94.9\%) & $196(95.6 \%)$ & 381 (95.3\%) & 0.73 \\
\hline Had cancer & $10(5.1 \%)$ & $9(4.4 \%)$ & $19(4.8 \%)$ & \\
\hline Aspirin use in last 20 & & & & \\
\hline $0-1$ year & $115(59.0 \%)$ & $115(56.1 \%)$ & $230(57.5 \%)$ & 0.19 \\
\hline 1 to $<5$ years & $40(20.5 \%)$ & $49(23.9 \%)$ & $89(22.3 \%)$ & \\
\hline 5 to $<10$ years & $16(8.2 \%)$ & $8(3.9 \%)$ & $24(6.0 \%)$ & \\
\hline $10+$ years & 24 (12.3\%) & 33 (16.1\%) & 57 (14.3\%) & \\
\hline
\end{tabular}


Table 1 Continued

\begin{tabular}{|c|c|c|c|c|}
\hline Characteristic & Placebo (N = 195) & Selenium ( $N=205)$ & Total $(\mathrm{N}=400)$ & P value* \\
\hline \multicolumn{5}{|c|}{ Non-steroidal anti-inflammatory drug use in last 20 years } \\
\hline 0 to $<1$ year & $176(90.3 \%)$ & $176(85.9 \%)$ & $352(88.0 \%)$ & 0.03 \\
\hline 1 to $<5$ years & $9(4.6 \%)$ & $23(11.2 \%)$ & $32(8.0 \%)$ & \\
\hline 5 to $<10$ years & $3(1.5 \%)$ & $4(2.0 \%)$ & $7(1.8 \%)$ & \\
\hline $10+$ years & $7(3.6 \%)$ & $2(1.0 \%)$ & $9(2.3 \%)$ & \\
\hline \multicolumn{5}{|l|}{ Clinic } \\
\hline Phoenix & $142(72.8 \%)$ & $148(72.2 \%)$ & $290(72.5 \%)$ & 0.33 \\
\hline Colorado & $43(22.1 \%)$ & $48(23.4 \%)$ & $91(22.8 \%)$ & \\
\hline Western New York & $3(1.5 \%)$ & $0(0.0 \%)$ & $3(0.8 \%)$ & \\
\hline Tucson & 7 (3.6\%) & $9(4.4 \%)$ & $16(4.0 \%)$ & \\
\hline
\end{tabular}

*t-test or analysis of variance for continuous variables, $\chi^{2}$ categorical variables. †From Arizona Food Frequency Questionnaire.

‡From baseline plasma.

participants for assessment using the mOGTT method of Cobelli et al. ${ }^{19}{ }^{20}$ Briefly, an 8-sample, 3-hour mOGTT was performed for each participant. The test was commenced after an overnight fast with $75 \mathrm{~g}$ oral dose of glucose, followed by intravenous blood draws at $0,10,20,30$, $60,90,120$ and $180 \mathrm{~min}$. Glucose concentrations were measured as described above.

Measurement of plasma Se concentrations were performed with the AAnalyst 600 atomic absorption spectrometer (Perkin-Elmer, Norwalk, Connecticut, USA) with a THGA graphite furnace with Zeeman background correction and a Se electrodeless discharge lamp. Each participant's samples were diluted with matrix modifiers containing $0.01 \%$ nickel nitrate hexahydrate and $0.0043 \%$ magnesium nitrate hexahydrate in $0.4 \%$ nitric acid and $0.2 \%$ triton X-100. Calibration standards were prepared using the method of additions. Known standards were included for every 10 samples, and each sample was run in triplicate.

Collection of sociodemographic, dietary and medical history data was performed using self-administered questionnaires on randomization to the Selenium Trial. The Arizona Food Frequency Questionnaire was employed for dietary data ascertainment and has been described in detail elsewhere. ${ }^{21}$

\section{Statistical analyses}

Means, SD and other summary statistics for the overall population and by Se and placebo group were calculated using standard statistical methods. Univariate comparisons by treatment used t-tests or analysis of variance for normally distributed variables. For non-normally distributed variables, univariate comparisons used non-parametric methods. Outcome variables, HOMA2- $\% \beta$ and HOMA2- $\% \mathrm{~S}$ were calculated using the HOMA2 API Oxford University 2004 as described above. Change in outcome variables, HOMA2- $\% \beta$ and HOMA2- $\% \mathrm{~S}$ was calculated by subtracting pre-measurements from post-measurements. Outcome difference variables were $\log$ transformed to achieve normality if necessary. Difference variables were modeled using linear regression on $\mathrm{Se} /$ placebo group and adjusting for baseline HOMA2 measurements, age, and gender. OGTT was log transformed and then analyzed using a linear mixed model approach controlling for correlations between multiple observations per participant. All analysis were performed using Stata V.15.0.

\section{RESULTS}

Tables 1 and 2 present the baseline characteristics of the participants in the Se and placebo arms for the HOMA subgroup and the OGTT subgroup, respectively.

As reported in the primary analysis of the Selenium Trial, the two arms were generally well balanced in regard to participant composition, with no meaningful differences by age, BMI, dietary intake of Se, gender, race or ethnicity, smoking status, medical history of diabetes or cancer, aspirin use, or clinic site. However, within the HOMA subgroup, there was a statistically significant difference in supplemental Se intake at baseline $(\mathrm{p}=0.03)$, non-steroidal anti-inflammatory drug use $(\mathrm{p}=0.03)$, and education $(\mathrm{p}=0.04)$, while education was the only variable that differed for the OGTT subgroup $(\mathrm{p}=0.04)$.

Table 3 shows the baseline (pre-supplementation) and follow-up results for HOMA2- $\% \beta$ and HOMA2- $\%$ S, after a mean of 2.9 years of supplementation with Se or placebo.

No statistically significant differences were observed for any of the assessments, with a mean change in HOMA2- $\% \beta$ of $3.1 \pm 29.8$ for those who were in the placebo group and 3.1 \pm 24.0 for those in the Se treatment group $(\mathrm{p}=0.99)$. Similar non-significant results were observed for change in HOMA2- $\% \mathrm{~S}(\mathrm{p}=0.40)$. A comparison of changes in HOMA2- $\% \beta$ and HOMA2- $\% \mathrm{~S}$ stratified by age and gender is presented in table 4; no statistically significant relationship between Se supplementation and either outcome measure was observed within any stratum. 
Table 2 Baseline characteristics of oral glucose tolerance test study participants

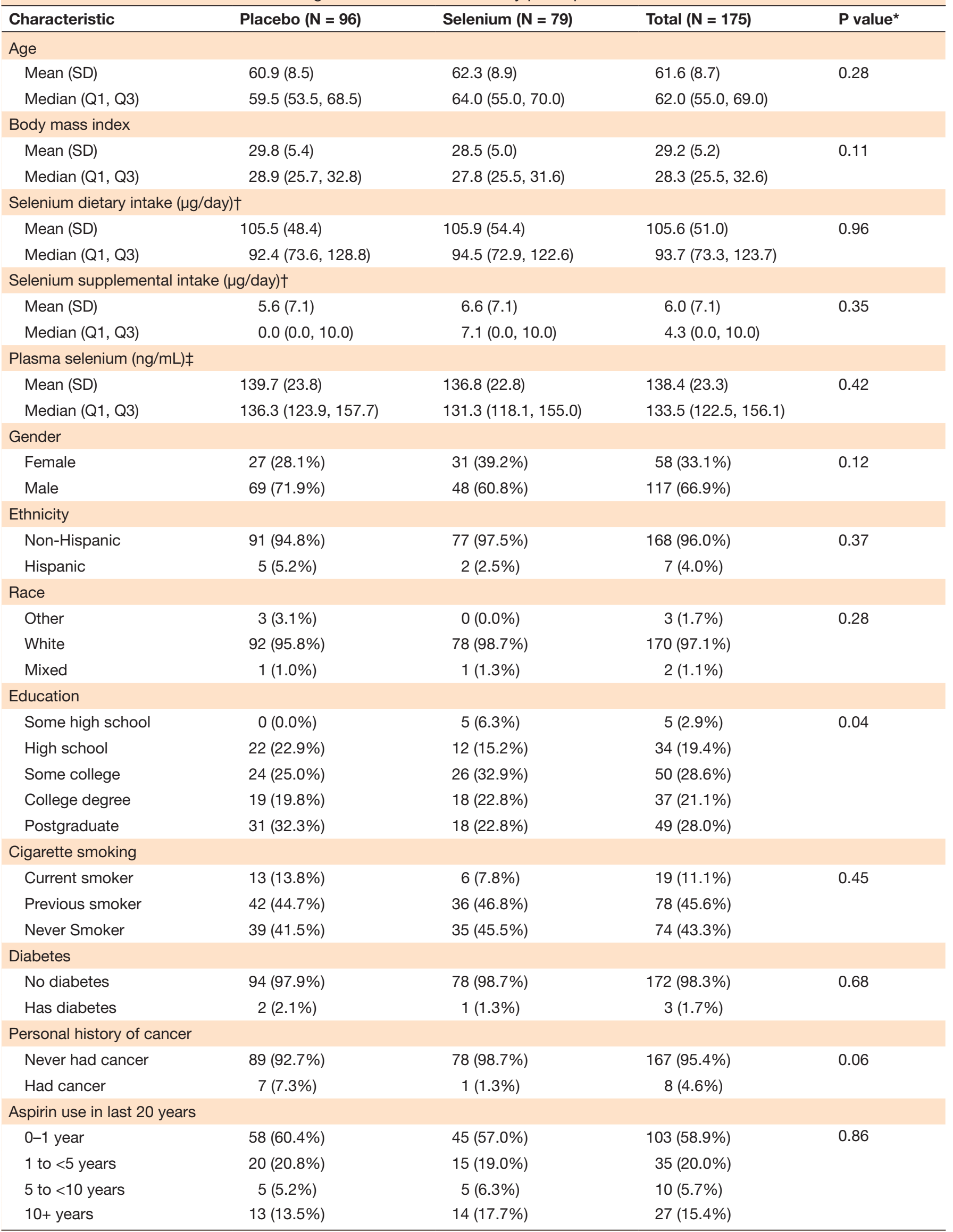

Continued 


(1)

Table 2 Continued

\begin{tabular}{|c|c|c|c|c|}
\hline Characteristic & Placebo $(\mathrm{N}=96)$ & Selenium $(\mathbf{N}=\mathbf{7 9})$ & Total $(\mathrm{N}=175)$ & P value* \\
\hline \multicolumn{5}{|c|}{ Non-steroidal anti-inflammatory drug use in last 20 years } \\
\hline 0 to $<1$ year & $93(96.9 \%)$ & $72(91.1 \%)$ & $165(94.3 \%)$ & 0.31 \\
\hline 1 to $<5$ years & $2(2.1 \%)$ & $3(3.8 \%)$ & $5(2.9 \%)$ & \\
\hline 5 to $<10$ years & $0(0.0 \%)$ & $2(2.5 \%)$ & $2(1.1 \%)$ & \\
\hline 10+ years & $1(1.0 \%)$ & $2(2.5 \%)$ & $3(1.7 \%)$ & \\
\hline \multicolumn{5}{|l|}{ Clinic } \\
\hline Phoenix & $69(71.9 \%)$ & $57(72.2 \%)$ & $126(72.0 \%)$ & 0.68 \\
\hline Colorado & $11(11.5 \%)$ & 7 (8.9\%) & $18(10.3 \%)$ & \\
\hline Tucson & $16(16.7 \%)$ & $14(17.7 \%)$ & $30(17.1 \%)$ & \\
\hline Mayo & $0(0.0 \%)$ & $1(1.3 \%)$ & 1 (0.6\%) & \\
\hline
\end{tabular}

*t-test or analysis of variance for continuous variables, $\chi^{2}$ categorical variables.

†From Arizona Food Frequency Questionnaire.

‡From baseline plasma.

Results for the OGTT assessment are presented in table 5, where glucose levels at baseline and 10, 20, 30, 60, 90, 120 and 180 min are compared between the Se and placebo groups after 2.9 years of follow-up.

Mean fasting glucose concentrations were statistically significantly higher among participants in the placebo group $(96.6 \pm 14.6 \mathrm{mg} / \mathrm{dL})$ than for those in the Se group $(92.3 \pm 12.0 \mathrm{mg} / \mathrm{dL} ; \mathrm{p}=0.04)$, and remained higher during the $10 \mathrm{~min}(\mathrm{p}=0.07)$ and $20 \mathrm{~min}$ intervals $(\mathrm{p}=0.05)$. At the five remaining time points ranging from 30 to 180 min, no differences in glucose concentrations were observed between the treatment groups. We additionally conducted an investigation of whether participants who had baseline glucose concentrations that were normal $(<100 \mathrm{mg} / \mathrm{dL})$, impaired $(\geq 100$ and $<126 \mathrm{mg} / \mathrm{dL})$, or diabetic $(\geq 126 \mathrm{mg} / \mathrm{dL})$ converted to any of these states

\begin{tabular}{|c|c|c|c|c|}
\hline & Placebo $(n=188)$ & Selenium (n=201) & Total $(n=389)$ & $P$ value \\
\hline \multicolumn{5}{|l|}{ HOMA2-\% $\beta$} \\
\hline \multicolumn{5}{|c|}{ Baseline \% beta cell function } \\
\hline Mean (SD) & 95.5 (29.6) & $97.6(32.0)$ & $96.6(30.9)$ & $0.51^{*}$ \\
\hline Median (Q1, Q3) & $94.0(77.0,112.5)$ & $91.9(76.9,112.4)$ & $92.6(77.0,112.4)$ & \\
\hline \multicolumn{5}{|c|}{ Post \% beta cell function } \\
\hline Mean (SD) & $98.6(37.2)$ & $100.6(34.0)$ & $99.6(35.6)$ & 0.57 \\
\hline Median (Q1, Q3) & $92.0(76.3,113.0)$ & $93.8(78.0,114.2)$ & $92.4(76.9,113.3)$ & \\
\hline \multicolumn{5}{|c|}{$\Delta \%$ beta cell function } \\
\hline Mean (SD) & $3.1(29.8)$ & $3.1(24.0)$ & $3.1(26.9)$ & 0.99 \\
\hline Median (Q1, Q3) & $-0.2(-11.0,10.5)$ & $1.5(-11.1,13.7)$ & $0.4(-11.0,12.4)$ & \\
\hline \multicolumn{5}{|l|}{ HOMA2-\%S } \\
\hline \multicolumn{5}{|c|}{ Baseline \% insulin sensitivity } \\
\hline Mean (SD) & $251.9(423.1)$ & $241.5(316.2)$ & $246.5(371.3)$ & $1.0 \dagger$ \\
\hline Median (Q1, Q3) & $140.8(87.1,289.1)$ & $146.9(77.4,284.5)$ & $144.3(83.5,284.9)$ & \\
\hline \multicolumn{5}{|c|}{ Post \% insulin sensitivity } \\
\hline Mean (SD) & $332.8(1603.3)$ & $241.0(393.2)$ & $285.4(1149.2)$ & 0.52 \\
\hline Median (Q1, Q3) & $131.3(74.0,270.4)$ & $136.1(79.4,284.4)$ & $133.9(79.0,282.1)$ & \\
\hline \multicolumn{5}{|l|}{$\Delta$ Insulin sensitivity } \\
\hline Mean (SD) & $80.9(1530.9)$ & $-0.5(223.2)$ & $38.9(1075.6)$ & 0.40 \\
\hline Median (Q1, Q3) & $-9.3(-56.7,31.4)$ & $-2.3(-53.9,43.5)$ & $-6.1(-54.1,34.2)$ & \\
\hline
\end{tabular}

*Student's t-test.

†Kruskal-Wallis test.

HOMA2-\% $\beta$, homeostatic model assessment of $\beta$-cell function; HOMA2-\%S, homeostatic model assessment of insulin sensitivity. 
during the trial (data not shown). We found that there were no differences by Se treatment as compared with placebo in the proportion of participants who changed to either an improved or worsening glucose concentration or stayed the same $(\mathrm{p}=0.86)$.

\section{CONCLUSIONS}

The results of this clinical trial of supplementation with $200 \mu \mathrm{g} /$ day Se versus placebo demonstrated no effect of Se treatment on measures of insulin action or secretion. These findings lend important evidence that Se supplementation for up to 3 years in older individuals does not elicit diabetogenic effects in humans and are in agreement with the majority of data from clinical trials of Se. In a comparison of HOMA2- $\% \beta$ and HOMA2- $\%$ S, no differences were observed between the Se treatment group and the placebo arm after a mean of 2.9 years of supplementation. For OGTT, there were differences in fasting glucose concentrations, which were significantly higher at time zero among those in the placebo group than those who took Se. This trend remained up to the 20 min assessment, after which there were no differences between the two groups. Interestingly, the Uppsala Longitudinal Study of Adult Men reported a similar phenomenon finding in that the male participants with higher plasma Se levels displayed lower early insulin response in baseline intravenous glucose tolerance tests. ${ }^{22}$ However, the authors also found no significant differences in
OGTT or hyperinsulinemic euglycemic clamp among the men with higher plasma Se levels after 20 years of follow-up and thus considered the baseline early insulin response finding a consequence of multiple comparison testing. ${ }^{22}$ Regardless, placebo and Se treatment groups both had fasting glucose concentrations that, on average, were well within the normal range. These results indicate that further research regarding the potential for effects of Se on glucose and insulin metabolism is warranted.

The results of the present study for mOGTT in particular are reminiscent of the original hypothesis related to Se and diabetes, where it was proposed that Se might reduce risk for impaired insulin signaling. ${ }^{10}{ }^{11}$ The concept that Se may be a risk factor, rather than protective, for diabetes gained momentum after the publication of secondary analyses of the Nutritional Prevention of Cancer (NPC) Trial. ${ }^{4}$ In that work, researchers compared the proportion of those with T2D who were randomized to the Se group to those in the placebo group after having been supplemented for an average of 7.7 years and found a statistically significantly increased risk for T2D among those who received $200 \mu \mathrm{g} /$ day Se, with a HR and $95 \%$ CI of 1.55 (1.03 to 2.33). ${ }^{4}$ SELECT was the next large clinical trial to report findings among the 35 000 male participants who were part of the trial to ascertain whether $200 \mu \mathrm{g} /$ day of Se supplementation could reduce the risk of prostate cancer. ${ }^{3}$ In this trial, no significant differences between the intervention and placebo

Table 4 Comparison of change in HOMA2-\% $\beta$ and HOMA2-\%S before and after selenium supplementation, stratified by sex and age

\begin{tabular}{llrrrrrr}
\hline & & N & Average & SD & Max & Min & P value \\
\hline HOMA2-\% $\boldsymbol{\beta}$ & & & & & & & \\
Female & Placebo & 57 & -2.7 & 17.2 & 30.9 & -56.4 & $0.08^{*}$ \\
& Selenium & 73 & 4.0 & 23.6 & 102.3 & -61.0 & \\
Male & Placebo & 131 & 5.6 & 33.6 & 202.6 & -67.2 & 0.41 \\
& Selenium & 128 & 2.5 & 24.3 & 90.3 & -65.0 & \\
Age $<63$ & Placebo & 87 & 1.8 & 33.3 & 202.6 & -67.2 & 0.86 \\
& Selenium & 95 & 2.6 & 24.2 & 90.3 & -65.0 & \\
Age $\geq 63$ & Placebo & 101 & 4.2 & 26.5 & 161.7 & -52.2 & 0.84 \\
& Selenium & 106 & 3.5 & 23.9 & 102.3 & -61.0 & \\
HOMA2-\%S & & & & & & & \\
Female & Placebo & 57 & -58.6 & 631.7 & 2173.5 & -4102.8 & $0.53 \dagger$ \\
& Selenium & 73 & -4.3 & 194.9 & 656.0 & -707.1 & \\
Male & Placebo & 131 & 141.6 & 1785.2 & 20345.5 & -731.5 & 0.50 \\
& Selenium & 128 & 1.7 & 238.6 & 1483.6 & -1165.7 & \\
Age $<63$ & Placebo & 87 & 170.7 & 2235.4 & 20345.5 & -4102.8 & 0.99 \\
& Selenium & 95 & 2.0 & 288.1 & 1483.6 & -1165.7 & \\
Age $\geq 63$ & Placebo & 101 & 3.6 & 268.1 & 2173.5 & -731.5 & 0.21 \\
& Selenium & 106 & -2.8 & 143.5 & 427.8 & -707.1 & \\
\hline
\end{tabular}

*Student's t-test.

†Kruskal-Wallis test.

HOMA2-\% $\beta$, homeostatic model assessment of $\beta$-cell function; HOMA2-\%S, homeostatic model assessment of insulin sensitivity. 
Table 5 Comparison of oral glucose tolerance test results for selenium and placebo groups

\begin{tabular}{|c|c|c|c|c|}
\hline $\begin{array}{l}\text { Glucose concentrations } \\
\text { (mg/dL) }\end{array}$ & Placebo (N=96) & Selenium ( $N=79$ ) & Total $(\mathrm{N}=175)$ & P value* \\
\hline \multicolumn{5}{|l|}{ Fasting } \\
\hline Mean (SD) & $96.6(14.6)$ & $92.3(12.0)$ & $94.7(13.6)$ & \multirow[t]{2}{*}{0.04} \\
\hline Median (Q1, Q3) & $94.0(87.0,102.5)$ & $91.0(83.0,101.0)$ & $94.0(85.0,101.0)$ & \\
\hline \multicolumn{5}{|l|}{$10 \mathrm{~min}$} \\
\hline Mean (SD) & 116.7 (20.3) & $111.4(17.4)$ & $114.3(19.2)$ & \multirow[t]{2}{*}{0.07} \\
\hline Median (Q1, Q3) & $111.5(103.0,127.0)$ & $112.0(103.0,122.0)$ & $112.0(103.0,125.0)$ & \\
\hline \multicolumn{5}{|l|}{$20 \mathrm{~min}$} \\
\hline Mean (SD) & $144.0(25.5)$ & $136.7(21.4)$ & $140.7(24.0)$ & \multirow[t]{2}{*}{0.05} \\
\hline Median (Q1, Q3) & $143.0(125.0,160.0)$ & $135.0(121.0,149.0)$ & $137.0(122.0,155.0)$ & \\
\hline \multicolumn{5}{|l|}{$30 \mathrm{~min}$} \\
\hline Mean (SD) & $161.1(32.1)$ & $155.2(27.9)$ & $158.4(30.3)$ & \multirow[t]{2}{*}{0.21} \\
\hline Median (Q1, Q3) & $154.0(136.0,185.0)$ & $156.0(134.0,169.0)$ & $154.5(135.0,177.0)$ & \\
\hline \multicolumn{5}{|l|}{$60 \mathrm{~min}$} \\
\hline Mean (SD) & $174.0(53.5)$ & $168.5(42.4)$ & $171.5(48.8)$ & \multirow[t]{2}{*}{0.46} \\
\hline Median (Q1, Q3) & $172.0(132.5,212.5)$ & $166.0(135.0,201.0)$ & $169.0(133.0,209.0)$ & \\
\hline \multicolumn{5}{|l|}{$90 \mathrm{~min}$} \\
\hline Mean (SD) & $155.6(53.8)$ & $151.5(44.4)$ & $153.7(49.7)$ & \multirow[t]{2}{*}{0.59} \\
\hline Median (Q1, Q3) & $146.0(121.0,192.0)$ & $145.0(120.0,186.0)$ & $145.0(121.0,190.0)$ & \\
\hline \multicolumn{5}{|l|}{$120 \mathrm{~min}$} \\
\hline Mean (SD) & $132.2(49.8)$ & $129.4(41.4)$ & $130.9(46.1)$ & \multirow[t]{2}{*}{0.70} \\
\hline Median (Q1, Q3) & $122.0(95.0,168.0)$ & $119.0(98.0,161.0)$ & $120.0(97.0,161.0)$ & \\
\hline \multicolumn{5}{|l|}{$180 \mathrm{~min}$} \\
\hline Mean (SD) & $86.6(38.3)$ & $78.7(31.3)$ & $83.0(35.4)$ & \multirow[t]{2}{*}{0.15} \\
\hline Median (Q1, Q3) & $76.0(64.0,98.0)$ & $69.5(58.0,94.0)$ & $74.0(59.0,97.0)$ & \\
\hline
\end{tabular}

*Student's t-test.

groups were observed for T2D, with a HR $(95 \% \mathrm{CI})$ of 1.07 (0.94 to 1.22$).^{2}$ For the parent trial of the present work, participants were supplemented with $200 \mu \mathrm{g} /$ day Se for a mean of 2.9 years, and no overall effect of Se on the incidence of T2D was observed, with an OR (95\% CI) of $1.25(0.74-2.11){ }^{3}$ However, when conducting stratified analyses, a significant increased odds for T2D was observed for participants older than the median age of 63 years (OR 2.21; 95\% CI 1.04 to 4.67). ${ }^{3}$ In the present study, we stratified for age based on the same cutpoint of 63 years, but found no modification of effect for either HOMA estimate. For OGTT, we were unable to stratify by age due to the smaller sample size of that subgroup.

The results of the present study and from clinical trials overall differ from the general findings from observational studies, the majority of which have demonstrated positive, direct associations between $\mathrm{Se}$ and the development of T2D. ${ }^{5-9}$ Further, within the Selenium Trial, we have previously reported the results of a cross-sectional investigation of baseline Se and prevalent T2D that revealed statistically significantly increased odds of T2D among those who had the highest concentrations of blood Se levels, with an OR (95\% CI) of 1.77 (1.16 to 2.71) for those in the highest tertile of Se versus the lowest. ${ }^{23}$ The difference in the findings for the observational study as compared with the clinical trial results suggests several possibilities. These include reverse causation, whereby the pathology of T2D affects Se concentrations, or the presence of residual confounding in the observational studies.

First, if Se is a causal agent for T2D, it would be expected that supplementation with this agent would result in a markedly increased rate of development of T2D in those in the treatment group as compared with the placebo group, which did not occur in the SELECT or Selenium Trial. ${ }^{23}$ While a significantly increased risk of T2D was found with supplementation in the NPC Trial, it is possible that this finding was due to chance given the conflicting results from larger clinical trials.

Another consideration is related to timing and duration of Se exposure. In the clinical trials discussed above, supplement or placebo were generally administered to older participants for 5 years or less. In contrast, the observational studies may reflect longer term dietary exposure and as such it may be that higher consumption of Se throughout the lifespan is associated with risk 
for insulin dysregulation, while supplementation for a comparatively brief period is not. Another possibility is that there is a third factor that affects both Se status and risk of T2D that is not adequately controlled in observational studies. Most of the published work, including our own cross-sectional analyses, ${ }^{23}$ has included adjusted models for key variables such as BMI, age, and dietary intake of specific macronutrients. This suggests that there may be an as-yet unidentified confounding variable or that there is residual confounding that has not been completely accounted for in adjusted models and which can have a substantial effect on measures of association in observational association studies. ${ }^{24}$ If this is the case, the results from clinical trials must carry more weight when evaluating whether there is truly a causal relationship between Se and insulin resistance or T2D.

The strengths of the present work include the study design and the measured outcomes for insulin resistance, including HOMA2-\% $\beta$, HOMA2-\%S and mOGTT. This was a clinical trial of Se for the prevention of colorectal adenoma for which carefully annotated data were collected for the participants and which in turn allowed us to investigate the role of Se in insulin resistance in a prospective manner. The treatment and placebo arms of the trial were well-balanced for variables that may affect the results of observational studies. Thus, causality can be assessed with more rigor than in cross-sectional analyses. In addition, a majority of large studies have relied on self-reports of T2D and/or use of diabetes medication to assess the endpoint. We specifically designed this substudy to capture biomarkers for insulin resistance in order to aid in elucidation of a potential mechanism of action. Nonetheless, there were limitations to the work that must be acknowledged. These include the relatively small sample size as compared with the parent trial overall, which for OGTT prevented further exploration of potential effect modification by age, gender, and race or ethnicity. Although there was sufficient statistical power to detect a statistically significant difference between the two treatment groups, there remains a possibility that the null findings were due to the comparatively small sample size.

In conclusion, the results of clinical trial do not support a causal role for Se in the development of insulin resistance or T2D as shown by the lack of differences in HOMA2-\% $\beta$ or HOMA2-\% $\mathrm{S}$ between groups who received Se or placebo for an average of 2.9 years. In addition, fasting blood glucose concentrations were higher for those in the placebo group compared with those in the Se treatment group. Further clarification of whether there are individual characteristics such as genotype that may influence response to Se is warranted.

Contributors ETJ, PL, LJM, H-HSC, and ETJ were responsible for the initial design of the project, as well as data collection. C-HPH, ETJ, and KS were responsible for conducting the data analyses. NAE, JF, JAM, C-HPH, and KB made key contributions to the preparation of the manuscript, specifically in interpretation of the data. All authors participated in the writing of the manuscript and have approved it in its final form for submission.
Funding Financial Support: the National Cancer Institute Cancer Center Support Grant P30 CA023074, NIH/NCI R01CA151708 (ETJ), NIH/NCI P01 CA041108 (PL); NIH R01DK047396 (LJM) and NIH/NCI R01CA151708 (PL).

Competing Interests None declared.

Patient consent for publication Not required.

Ethics approval The University of Arizona Institutional Review Board (IRB) approved and oversaw the study protocol.

Provenance and peer review Not commissioned; externally peer reviewed.

Data sharing statement Data requests should be directed to ETJ at jacobse@ email.arizona.edu. Deidentified data included in this work may be obtained through an established process. A data request form will be supplied for completion and approval by the authors the manuscript. Reuse is permitted for reasonable, scientifically rigorous research.

Open access This is an open access article distributed in accordance with the Creative Commons Attribution Non Commercial (CC BY-NC 4.0) license, which permits others to distribute, remix, adapt, build upon this work non-commercially, and license their derivative works on different terms, provided the original work is properly cited, appropriate credit is given, any changes made indicated, and the use is non-commercial. See: http://creativecommons.org/licenses/by-nc/4.0/.

\section{REFERENCES}

1. Clark LC, Combs GF, Turnbull BW, et al. Effects of selenium supplementation for cancer prevention in patients with carcinoma of the skin. A randomized controlled trial. Nutritional prevention of cancer Study Group. JAMA 1996;276:1957-63.

2. Lippman SM, Klein EA, Goodman PJ, et al. Effect of selenium and vitamin $E$ on risk of prostate cancer and other cancers: the Selenium and Vitamin E Cancer Prevention Trial (SELECT). JAMA 2009;301:39-51.

3. Thompson PA, Ashbeck EL, Roe DJ, et al. Selenium supplementation for prevention of colorectal adenomas and risk of associated type 2 diabetes. J Natl Cancer Inst 2016;108.

4. Stranges S, Marshall JR, Natarajan R, et al. Effects of long-term selenium supplementation on the incidence of type 2 diabetes. Ann Intern Med 2007;147:217-23.

5. Zhang H, Yan C, Yang Z, et al. Alterations of serum trace elements in patients with type 2 diabetes. J Trace Elem Med Biol 2017;40:91-6.

6. Wei J, Zeng C, Gong Q-yi, et al. The association between dietary selenium intake and diabetes: a cross-sectional study among middle-aged and older adults. Nutr J 2015;14:18.

7. Laclaustra M, Navas-Acien A, Stranges S, et al. Serum selenium concentrations and diabetes in U.S. Adults: National Health and Nutrition Examination Survey (NHANES) 2003-2004. Environ Health Perspect 2009;117:1409-13.

8. Bleys J, Navas-Acien A, Guallar E. Serum selenium and diabetes in U.S. Adults. Diabetes Care 2007;30:829-34.

9. Stranges S, Galletti F, Farinaro E, et al. Associations of selenium status with cardiometabolic risk factors: an 8-year follow-up analysis of the Olivetti heart study. Atherosclerosis 2011;217:274-8.

10. McNeill JH, Delgatty HLM, Battell ML. Insulinlike effects of sodium selenate in streptozocin-induced diabetic rats. Diabetes 1991;40:1675-8.

11. Mueller AS, Pallauf J. Compendium of the antidiabetic effects of supranutritional selenate doses. in vivo and in vitro investigations with type II diabetic db/db mice. J Nutr Biochem 2006;17:548-60.

12. Papp LV, Lu J, Holmgren A, et al. From selenium to selenoproteins: synthesis, identity, and their role in human health. Antiox Redox Signal 2007;9:775-806.

13. Rayman MP, Stranges S. Epidemiology of selenium and type 2 diabetes: can we make sense of it? Free Rad Biol Med 2013;65:1557-64.

14. McClung JP, Roneker CA, Mu W, et al. Development of insulin resistance and obesity in mice overexpressing cellular glutathione peroxidase. Proc Natl Acad Sci 2004;101:8852-7.

15. Loh K, Deng H, Fukushima A, et al. Reactive oxygen species enhance insulin sensitivity. Cell Metab 2009;10:260-72.

16. Thompson P, Roe DJ, Fales L, et al. Design and baseline characteristics of participants in a phase III randomized trial of celecoxib and selenium for colorectal adenoma prevention. Cancer Prev Res 2012;5:1381-93.

17. Sawilowsky SS. New effect size rules of thumb. J Mod App/ Stat Meth 2009;8:597-9.

18. Wallace TM, Levy JC, Matthews DR. Use and abuse of HOMA modeling. Diabetes Care 2004;27:1487-95. 
19. Cobelli C, Toffolo GM, Dalla Man C, et al. Assessment of beta-cell function in humans, simultaneously with insulin sensitivity and hepatic extraction, from intravenous and oral glucose tests. Am J Physiol Endocrinol Metab;2007;293:E1-E15.

20. Dalla Man C, Campioni M, Polonsky KS, et al. Two-hour sevensample oral glucose tolerance test and meal protocol: minimal model assessment of beta-cell responsivity and insulin sensitivity in nondiabetic individuals. Diabetes 2005;54:3265-73.

21. Ritenbaugh C, Aickin M, Taren D, et al. Use of a food frequency questionnaire to screen for dietary eligibility in a randomized cancer prevention phase III trial. Cancer Epidemiol Biomarkers Prev 1997:6:347-54

22. Gao H, Hägg S, Sjögren $P$, et al. Serum selenium in relation to measures of glucose metabolism and incidence of type 2 diabetes in an older Swedish population. Diabet Med 2014;31:787-93.

23. Kohler LN, Florea A, Kelley CP, et al. Higher plasma selenium concentrations are associated with increased odds of prevalent type 2 diabetes. J Nutr 2018;148:1333-40.

24. Liang W, Zhao Y, Lee AH. An investigation of the significance of residual confounding effect. Biomed Res Int 2014;2014:1-6. 